\title{
Advanced technologies for shoe sole production
}

\author{
Tatjana Spahiu ${ }^{1, *}$, Henrique Almeida ${ }^{2,3}$, Rita M. T. Ascenso ${ }^{2,3}$, Liliana Vitorino ${ }^{2}$, and \\ Anabela Marto ${ }^{2,3,4}$ \\ ${ }^{1}$ Faculty of Mechanical Engineering, Textile and Fashion Department, Polytechnic University of \\ Tirana, Albania. \\ ${ }^{2}$ School of Technology and Management, Polytechnic Institute of Leiria, Leiria, Portugal. \\ ${ }^{3}$ Computer Science and Communication Research Centre, Polytechnic Institute of Leiria, Leiria, \\ Portugal. \\ ${ }^{4}$ Information Systems and Computer Graphics, Institute for Systems and Computer Engineering, \\ Technology and Science (INESC TEC), Porto, Portugal.
}

\begin{abstract}
Advanced technologies for modelling and production are an important part in the whole process of product manufacturing. These advancements have changed the way of product development and play an important role in customization. In the footwear industry, as in any other industry, the use of these technologies is widely spread. Footwear comfort is one of the main selection criteria for purchase. Considering this fact, a case study of different steps for shoe designing according to individual foot shape will be presented. Taking into consideration the aesthetics of the sole and in a more sustainable view, through topological optimization reducing of material wastage for sole production will be presented. By means of the topological optimization in the shoe design process, sole optimization is realized. As a part of personalization, feet's plantar pressure maps taken from 1 participant gave a better explanation of weight distribution of each foot. Following, sole personalization according the plantar pressure maps for each foot gives the possibility to obtain the best least material design according to the feet's pressure while maintaining biomechanical performance.
\end{abstract}

\section{Introduction}

Footwear is a garment worn by feet but with high requirements regarding fit and comfort compared to the other type of garments. The feet support us for a long time sowe need to show a great care. As the first step of footwear production, shoes last plays an important role in the fit and comfort and using the right last and materials, shoes with the right fit and comfort are produced. Insoles are one of the most important parts of the footwear as they are in direct contact with the foot. An insole helps to correct any mal-alignment problems and makes it easier to walk comfortably, run easily, and avoid injury [1]. Applications of $3 \mathrm{D}$ printing in the shoes industry are of great interest. A researcher conducted a study by

*Correspondingauthor:tspahiu@fim.edu.al 
wearing a customized 3D-printed single-sided lateral wedge insole and demonstrated gait rehabilitation [2]. Despite the shape, different materials used for insole production play an important role by affecting ankle dorsiflexion and plantarflexion and maximum knee flexion during walking [3]. Researches done from several authors have concluded that production of anatomical insoles by 3D printing is cost effective and feasible at the industrial level [4], reducing pressure [5] and through the use of 3D scanning making it an affordable option for footwear [6]. With CAD tools designed by a group of authors it is possible to reduce time, increase sole qualityand alsofunctionality. Moreover, 3D printing technology offers new possibilities for manufacturing of complex geometries [7]. The sole is usually made of leather, vulcanized rubber, TPR (thermoplastic rubber), polyurethanes, TPU (thermoplastic polyurethanes) or EVA (ethylene vinyl acetate) [8]. Sole hardness plays an import role in footwear comfort. The tests conducted on the relationship between foot pressure distribution and sole hardness found a great impact on the shoe comfort [9]. Due to irregular geometries and complex material properties of soles, computational modelling as FEM techniques help to study the foot-footwear interaction, where personalization of insoles is more important to reduce peak plantar pressure than the stiffness of the material used for insole production [10]. By mean of reverse engineering, a produced shoe can be used to investigate and suggest improvements of material allocation within the midsole [11]. Results taken from finite element simulations of insole and midsolelayers made from various foam materials as the case of sport shoes optimize the distribution of the elastic modulus in the midsole to improve operability [12] aiming to reduce the Ground Reaction Forces [13]. Moreover, these applications help in controllingsole properties by using lattice structures [14] and through the combination of mechanical meta-material structures to create personalized soles [15] and shoes by 3D printing [16]. Lattice structures are generated by some CAD software's, butthey may present difficulties in the operationalization of the design process. To avoid this, researchers have developed an interface in Visual Basic programming language which is based on the API functions in SolidWorks. This can help to automatically generate complex models of lattice structures [17] and using finite element analysis method to investigate the mechanical properties of each configuration of the lattice structure [18] and produce by 3D printing and investment casting [19]. Another innovative technique as Constrained Free Form Deformation and 3D scanning have shown to have a great impact by accelerating the process of shoe development and production [20]. Since footwear is the interface between the foot and the floor which has relevance for each person, the investigation, understanding and management of foot biomechanics through pressure measurement is mandatory for footwear design. Foot plantar pressure is the pressure field that acts between the foot and the support surface during everyday locomotor activities. Plantar pressure assessment alone is not enough to thoroughly investigate foot biomechanics. Plantar pressure, however, has great potentialities in the field of research and footwear customization [21].

\section{A Case Study - Shoe Redesign with Topological Optimization Based on Biomechanical Data}

To demonstrate the potential of combining biomechanics with several digital and physical technologies, a shoe design case study is presented. It focuses on a sole model that was previously designed in Shoemaker software as depicted in figure 1.In a previous work the numerical simulation of sole models was realized. In this case without taking into considerations the feet pressure maps [22]. But considering the biomechanical factor that each person presents asymmetric behaviourbetween both sides, the biomechanical study began with the definition of the feet's plantar pressure in order to understand the differences between both the left and right foot. 

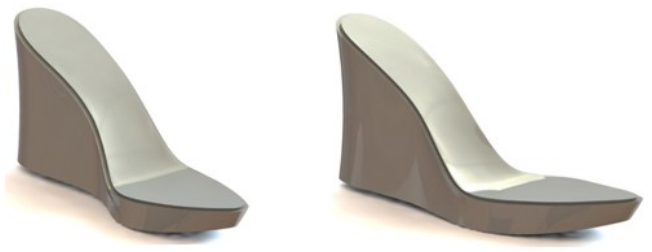

Fig. 1.The left and right side shoe models.

In figure 2 is illustrated the scanning procedure and the obtained plantar pressure plot and values. The hardware system used is a high speed $2 \mathrm{~m}$ Hi-End footscan ${ }^{\circledR}$ system and the software used is the Footscan ${ }^{\circledR}$ 9, both from RSSscan Systems. The test was repeated 3 times for the patient.

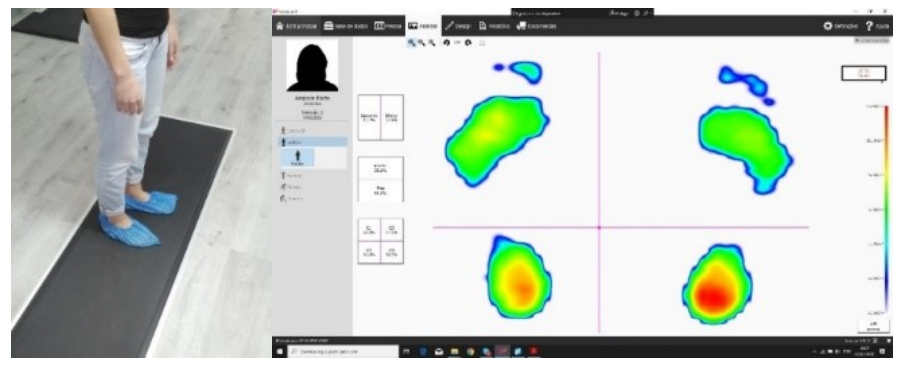

Fig. 2.Scanning of the feet's plantar pressure and obtained results ofpressure plot.

The next step consisted in creating the curves for the generation of the surfaces of the pressure areas on the soles for the definition of the loading conditions for both the structural simulations and the topological optimizations. With the aid of CAD software (SolidWorks 2019), the plantar pressure plot was imported into the software and the necessary scaling of the image was performed in order to correspond with the patient's shoe size of 37. Also, both the left and right CAD soles were orientated to correspond both feet with the plantar pressure plot. Figure 3 illustrates the orientation of both soles and the pressure curves used to create the surface areas according to the plantar pressure plot.
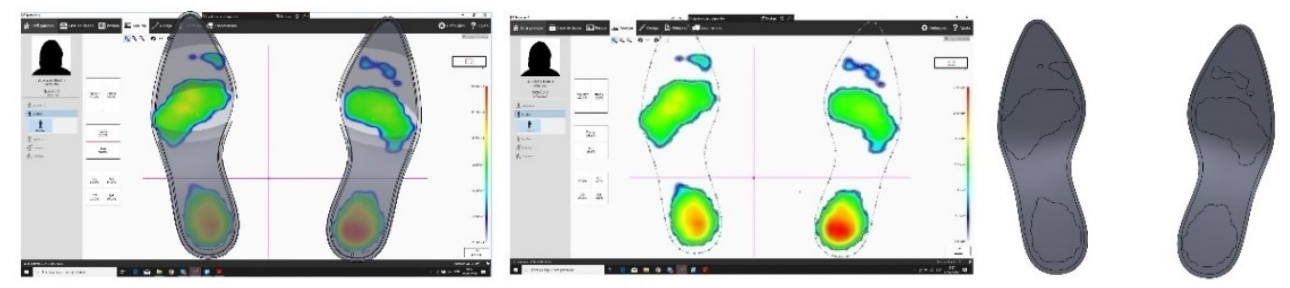

Fig.3. Image import, curve definition and surface definition of the pressure areas.

To begin the engineering design phase, the first step consisted in performing the structural simulations in order to validate the mechanical performance of the designed sole. The patient's data was used in the simulations to define the loading conditions for both the left and right side. The previous designed pressure areas along with the values of the pressure plots and distributed weight is presented in figure 4. The patient presents a weight of $51 \mathrm{~kg}$ with a difference of $5.6 \%$ in weight distribution between both the left and right side. The fixed constraints were defined on the lower surface of both sole models which were meshed with the same mesh size in order to perform an adequate comparison between both models. Considering that Additive Manufacturing is to be used for the fabrication of 
the shoe soles in an affordable system to guarantee that the price of both soles is similar when compared to other regular designed shoes in the market, the material selected was TPU 95A from Ultimaker. The results of the structural simulations are presented in figure 5 and demonstrate that the designed shoe can withstand the patient's weight. Alsoit is visible the influence of the patient's weight distribution in both the stress and displacement results.
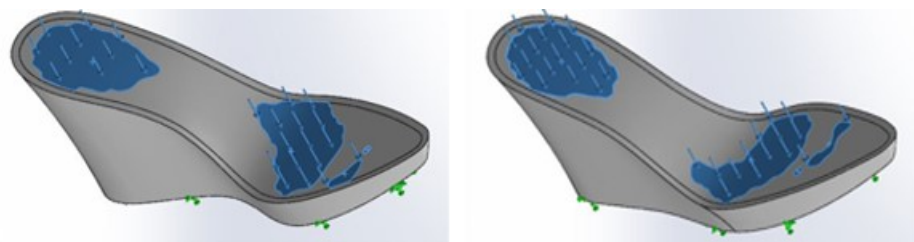

Fig. 4.Loading and constraint conditions for both shoe soles.

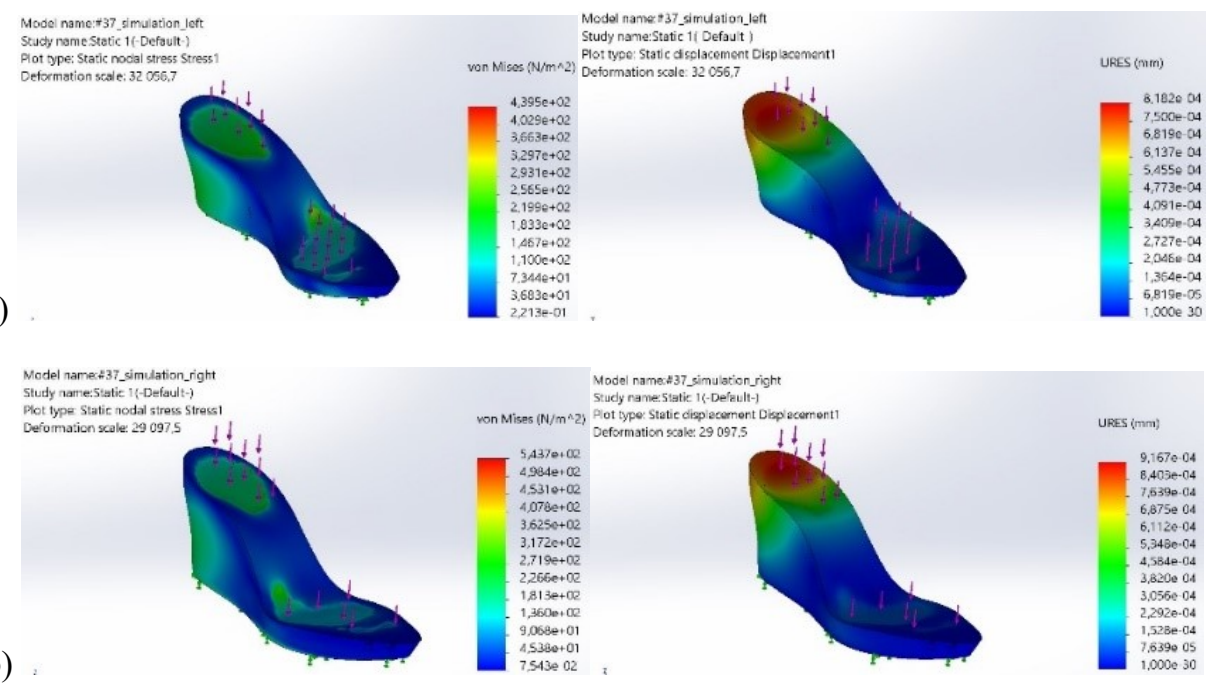

Fig. 5.Stress and displacement results for both feet: a) left and b) right side.

After performing the structural simulations and validating both the design and material selection, the topological optimisation was then performed based on a mathematical method that optimizes the material layout within a given design space, for a given set of loads, boundary conditions and constraints with the goal of maximizing the system performance. The material percentage reduction for both soles was of $25 \%$ and the area selected in which the topological optimisation will occur is the centre core of the shoe sole. Figure 6 illustrates the initial model and then the optimised topological design of the centre cores for both the left and right sides. Similar, to the structural simulations, again the optimised designs also present differences between them.

a)
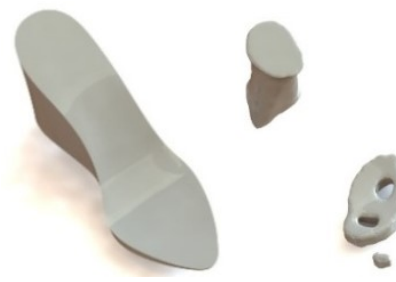

b)
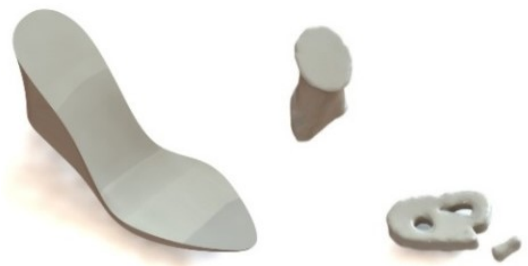

Fig. 6. Topological optimisation solutions of the centre sole for the a) left and b) right sides. 
Figure 7 illustrates the final topological optimized design for both the left and right shoe soles.From the work presented, it is possible to observe that engineering design schemes may be introduced in shoe design with an additional characteristic of structural simulations in order to predict the product's mechanical performance. To end the product design cycle, the following step is to produce both designs for consumer approval, which may suffer new iterations according to the consumer's preferences.
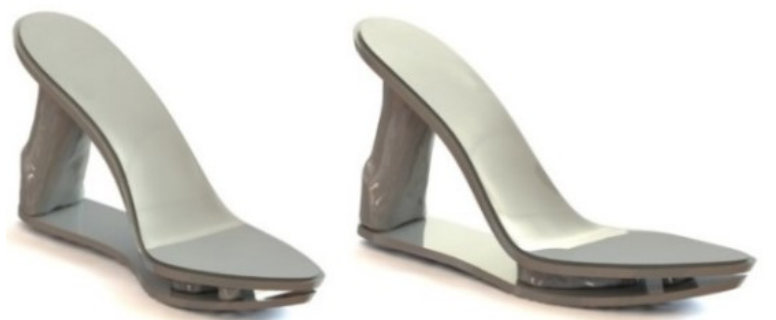

Fig. 7.The left and right side topological optimized shoe models.

\section{Conclusions}

Personalisation helps to improve the efficiency and reduce costs, by offering the companies a sustainable growth with differentiated products and services in a profitable manner. This is already possible due to the advanced digital and physical manufacturing systems and distribution technologies. In this paper, a case study focused on the design of a shoe design considering the biomechanical asymmetry of the consumer. After obtaining the 3D models and the biomechanical data, engineering design schemes was implemented, namely topological design. From the framework presented, it is possible to observe that engineering design schemes can be introduced in shoe design with an additional characteristic of structured simulations in order to predict the product's mechanical performance while maintaining a tailored aesthetic appearance and most important a biomechanical performance.

\section{References}

1 Y. Urabe, N. Maeda, S. Kato, H. Shinohara and J. Sasadai, J Phys Fitness Sports Med. 3, no. 4, 385-398, doi.org/10.7600/jpfsm.3.385, (2014).

2 H. Jin, R. Xu and J. Wang, Med Sci Monit. 25, 25:7720-7727, doi:10.12659/MSM.919400(2019).

3 R. Ozmanevra, S. Angin, I. H. Gunal and A. Elvan, Am Podiatr Med Assoc. 108, no. 5,390-396,doi.org/10.7547/16-175, (2018).

4 M. Davia-Aracil, J. J. Hinijo-Perez, A. Jimeno-Morenilla and H. Mora-Mora, Computers in Industry 95, 38-53, (2018).

5 S. Telfer, J. Pallari, J. Munguia, K. Dalgarno, M. McGeough and J. Woodburn, BMC Musculoskelet Disord. 13, no. 84, doi.org/10.1186/1471-2474-13-84, (2012).

6 A. S. Salles and D. E. Gyi, J Sports Sci. 31, no. 4: 442-450. doi:10.1080/02640414.2012.736629, (2013).

7 M. Davia-Aracil, A. Jimeno-Morenilla and F. Slas, Int J Adv Manuf Technol. 86, 34953516, doi.org/10.1007/s00170-016-8427-5, (2016).

8 S. S. Muthu, S. Limited and P. R. China, "The environmental impact of footwear and footwear materials," Handbook of footwear design and manufacture, A. Luximon, The 
Textile Institute and Woodhead Publishing, (2013).

9 X. Xie, Leather Footwear J. 18, no. 4, 249-258, doi: 10.24264/lfj.18.4.1, (2018).

10 J. T-M. Cheung. M. Zhang, Arch Phys Med Rehabi. 86, no. 2, 353-358, doi: 10.1016/j.apmr.2004.03.031, (2005).

11 D. Drougkas, E. Karatsis, M. Papagiannaki, S. Chatzimoisiadis, F. Arabatzi, S. Maropoulos and A. Tsouknidas, Appl Bionics Biomech, 9, doi.org/10.1155/2018/6520314, (2018).

12 K. Shimoyama, K. Seo, T. Nishiwaki, S. Jeong and S. Obayashi, J Sport Eng Technol. 225, no. 4, 179-188, doi.org/10.1177/1754337111414485, (2011).

13 M. R. Shariatmadari and R. English and G. Rothwell, "Finite Element Study into the Effect of Footwear Temperature on the Forces Transmitted to the Foot during QuasiStatic Compression Loading," IOP Conf. Series: Materials Science and Engineering, doi.org/10.1088/1757-899X/10/1/012126, (2010).

14 D. Guoying, T. Daniel and Z. Y. Fiona, "Design of Shoe Soles Using Lattice Structures Fabricated by Additive Manufacturing," 22nd International Conference on Engineering Design, Delft, The Netherlands, doi.org/10.1017/dsi.2019.76(2019).

15 D. J. Nogueira Amorim, T. Nachtigall and M. B. Alonso, "Exploring Mechanical MetaMaterial Structures through Personalised Shoe Sole Design," Symposium on Computational Fabrication (SCF '19), Pittsburgh, PA, USA, doi.org/10.1145/3328939.3329001 (2019).

16 T. R. Nachtigall, S. Wensveen, O. Tomico, P. Van Dongen, R. Wakkary and L. T. Van Noorden, "Towards Ultra Personalized 4D Printed Shoes,"CHI Conference on Human Factors in Computing Systems, Montreal QC Canada, doi.org/10.1145/3170427.3174369, (2018).

17 D. S. Nguyen, T. Tuan Tran, D. Kien Le and V. Than Le, "Creation of Lattice Structures for Additive Manufacturing in CAD Environment," International Conference on Industrial Engineering and Engineering Management, Bangkok, Thailand, doi: 10.1109/IEEM.2018.8607271 (2018).

18 D. S. Nguyen and T. A. Nguyen-Van, J. Korean Soc. Precis. Eng. 37, no. 4, $305-$ 318, doi.org/10.7736/JKSPE.019.100 (2020).

19 G. Maliris, I. T. Sarafis, T. Lazaridis, A. Varoutoglou and G. Tsakataras, "Random lattice structures. Modelling, manufacture and FEA of their mechanical response," IOP Conf. Series: Materials Science and Engineering 161, doi:10.1088/1757899X/161/1/012045(2016).

20 L. Orazi and B. Reggiani, Int J Adv Manuf Technol, 106, 2295-2303, doi.org/10.1007/s00170-019-04717-8, (2020).

21 F. T. Piller \&. M.Müller, Int. J. Comput. Integr. Manuf. 17, no. 7, 583-593, doi.org/10.1080/0951192042000273140,(2004).

22 T. Spahiu, E. Piperi, A. Ehrmann, H.A.Almeida, R.M.T.Ascenso, L.C.Vitorino,"3D Printing: An Innovative Technology for Customised Shoe Manufacturing",Progress in Digital and Physical Manufacturing, 171-180, doi.org/10.1007/978-3-030-29041-2_22, (2019). 